\section{AA-02 THE EXPRESSION OF AUTOANTIBODIES TO MITOCHONDRIA IN THE BLOOD OF PATIENTS WITH SLE}

${ }^{1}$ David S Pisetsky*, ${ }^{1}$ Diane M Spencer, ${ }^{2}$ Fariborz Mobarrez, ${ }^{2}$ Enrico Fuzzi, ${ }^{2}$ Elisabet Svenungsson. ${ }^{1}$ Department of Medicine, Duke University Medical Center; Medical Research Service, Durham VA Medical Center, Durham, North Carolina USA; ${ }^{2}$ Unit of Rheumatology, Department of Medicine, Solna, Karolinska Institutet, Karolinska University Hospital, SE-171, 76 Stockholm, Sweden

\subsection{6/lupus-2018-Ism.21}

Background Systemic lupus erythematosus (SLE) is a prototypic autoimmune disease characterized by the expression of antibodies to extracellular vesicles (EVs). These vesicles can arise from dead and dying cells and display nuclear and cytoplasmic molecules. Since EV preparations contain mitochondria, we performed experiments to test directly the binding of SLE antibodies to mitochondria.

Methods Mitochondria were prepared from mouse liver and immobilized on microtiter plates pre-coated with poly-l-lysine at $0.5 \mu \mathrm{g} / \mathrm{ml}$. Bound antibodies were detected using a peroxidase-conjugated anti-IgG reagent. To determine whether DNA contributed to the antigenicity of the mitochondria, the ability of DNA to inhibit binding was tested. The binding of sera from 211 SLE patients who met 1982 ACR criteria for classification was determined by ELISA and results compared with an ELISA for M2 antimitochondrial antibodies (AMA).

Results Using an ELISA assay with immobilized mitochondria, $60.2 \%$ of SLE sera showed positive responses defined as greater than two standard deviations above the mean of control sera. Samples were also analyzed using a commercial AMA ELISA (Euroimmun US, Morris Plains, New Jersey) for IgG antibodies to the M2 antigen. With this kit, $5.7 \%$ of the SLE samples tested positive. To determine the relationship of antibodies to anti-DNA, the ability of calf thymus (CT) DNA to inhibit binding to mitochondria was investigated for a subset of samples. In the ELISA, CT DNA at $50 \mu \mathrm{g} / \mathrm{ml}$ inhibited binding for 7 of 8 SLE plasmas, with inhibition ranging from 7.7\%-59.3\%.

Conclusion These results indicate that blood of patients with SLE contain antibodies to mitochondria. Among these antibodies, some may react to DNA as shown by the ability of soluble DNA to inhibit ELISA binding. While binding mitochondria, these antibodies differ from AMA found in primary biliary cholangitis since few reacted in the ELISA for M2 AMA. Since studies using flow cytometry have demonstrated that IgG positive EVs in the blood of patients contain mitochondria as shown by MitoTracker Deep Red, these results suggest that, like nuclei, mitochondria may be a subcellular organelle that can display autoantigenic determinants to form immune complexes in SLE.

\section{AA-03 NEURONAL-SURFACE P-ANTIGEN (NSPA), TARGET OF ANTI-RIBOSOMAL P AUTOANTIBODIES, IS EXPRESSED IN PROXIMAL TUBULE EPITHELIAL CELLS (PTEC): POTENTIAL ROLE IN LUPUS NEPHRITIS}

\footnotetext{
${ }^{1}$ Marcela Bravo-Zehnder, ${ }^{1}$ Patricia Gajardo, ${ }^{1}$ Daniela Valenzuela, ${ }^{1}$ Tomás Toledo ${ }^{1}$ Ángel Jurado, ${ }^{1}$ Fabián Segovia, ${ }^{2}$ Gonzalo Méndez, ${ }^{2}$ Carlos Vio, ${ }^{1}$ Loreto Massardo*, ${ }^{1,2}$ Alfonso González. ${ }^{1}$ Center for Cell Biology and Biomedicine (CEBICEM), Faculty of Medicine and Science, Universidad San Sebastián, Santiago, Chile; ${ }^{2}$ Center for Aging and Regeneration (CARE), Faculty of Biological Science, Pontificia Universidad Católica de Chile, Santiago, Chile
}

10.1136/lupus-2018-Ism.22
Background Patients with systemic lupus erythematosus (SLE) and anti-ribosomal P (anti-P) antibodies may present lupus psychosis, cognitive impairment and lupus nephritis (LN). Our group provided a molecular mechanism to explain the neuronal alterations in SLE identifying the neuronal-surface P-antigen (NSPA) as an anti-P target involved in the regulation of synaptic glutamatergic transmission (Bravo-Zehnder et al. A and $R$ 2015; Segovia-Miranda et al. $A$ and $R$ 2015). There is evidence suggesting that anti-P and anti-dsDNA antibodies have the potential to perturb the function of renal cells in addition to complement activation. The mechanism of anti-P association with lupus nephritis remains unclear. It is possible that direct anti-P interaction with cell surface components activates intracellular signalling pathways resulting in renal cell damage. As a first approximation to this hypothesis we study whether and where NSPA is expressed in the kidney.

Methods NSPA expression was assessed by RT-PCR, immunoblot and anti-NSPA immunohistochemistry in $\mathrm{C} 57^{\mathrm{WT}}$ mice, $\mathrm{B}$ galactosidase (B-gal) histochemistry staining in transgenic mice bearing a LacZ gene under the promotor of NSPA gene and anti-P staining in the surface of primary cultures of PTEC and the human kidney cell line HK2. NSPA polarized distribution was studied in Madin-Darby canine kidney (MDCK) cells transfected with NSPA-GFP expression plasmid. The pathogenic potential of anti-P antibodies was analyzed by immunizing $\mathrm{C} 57^{\mathrm{WT}}$ and NSPA ${ }^{\mathrm{KO}}$ mice with recombinant P0 ribosomal bearing $\left(\mathrm{PO}^{\mathrm{wt}}\right)$ or not the $\mathrm{P}$ epitope $\left(\mathrm{PO}^{\mathrm{tr}}\right)$ and testing anti-P and anti-dsDNA presence, proteinuria and renal pathology by histochemistry and electron microscopy.

Results NSPA is expressed in PTEC cells and display a mainly apical distribution in vivo and in vitro, including the transfected MDCK cells. Both $\mathrm{P}^{\text {wt }}$ and $\mathrm{P}^{\text {tr }}$ generated high levels of anti-P antibodies and some mice also developed anti-dsDNA antibodies. Mice presented mild proteinuria at 6 months of immunization. $\mathrm{C}_{5} 7^{\mathrm{WT}}$ and $\mathrm{NSPA}^{\mathrm{KO}}$ mice immunized with either $\mathrm{P}^{\mathrm{wt}}$ or $\mathrm{P} 0^{\text {tr }}$ also displayed perivenular lymphocytic infiltration, a mild renal pathology change. In addition, $\mathrm{NSPA}^{\mathrm{KO}}$ mice, even in absence of anti-dsDNA, showed mesangial electron dense-deposits with discrete effacement of foot processes.

Conclusions NSPA is expressed in both PTEC and polarized MDCK epithelial cells with a polarized distribution that precludes direct interaction with circulating anti-P antibodies, which might access to this target only after glomerular filtration. Circulating anti-P can associate with mild kidney damage independently of NSPA expression, at least in non-lupus prone mice.

Acknowledgements CONICYT Basal grant PFB12/2007 and FONDECYT Grant 1160513.

\section{AA-04 AUTOANTIBODIES TO M-PHASE PHOSPHOPROTEIN I (MPP-1: KIF20B) IN SYSTEMIC LUPUS ERYTHEMATOSUS}

${ }^{1}$ May Y Choi ${ }^{*}$, 'Eric Campbell, ${ }^{1}$ Ann Clarke, ${ }^{1}$ Michelle Jung, ${ }^{1}$ Claire Barber, ${ }^{2} Y$ van St Pierre, ${ }^{1}$ Marvin J Fritzler. 'University of Calgary, Cumming School of Medicine, Calgary, Alberta, Canada; ${ }^{2}$ Research Institute of the McGill University Health Centre, Montreal, Quebec, Canada

\subsection{6/lupus-2018-Ism.23}

Background M-phase phosphoprotein (MPP-1), also termed kinesin interacting protein (KIF20B), is a $210 \mathrm{kDa}$ protein that is highly expressed during cell division. Autoantibodies to MPP-1 were first described in approximately $25 \%$ of patients 